\title{
Lymphedema Management
}

National Cancer Institute

\section{Source}

National Cancer Institute. Lymphedema Management. NCI Thesaurus. Code C116697.

Any therapeutic technique that is aimed at reducing and/or preventing obstruction of the lymphatic system. 